\title{
Ludwig Burgmann
}

\section{Reformation oder Restauration? Zum Ehegüterrecht der Ecloga}

In seiner grundlegenden Darstellung der ,inneren“ Geschichte des griechisch-römischen Rechts hat Karl Eduard Zachariä von Lingenthal vom ehelichen Güterrecht der Ecloga als einem „ganz eigenthümlichen“ gesprochen und befunden, daß es vom justinianischen Recht „sehr wesentlich“ abweiche ${ }^{1}$. Diese Feststellung war außerordentlich folgenreich.

Zunächst für Zachariäs Periodisierungskonzept der griechisch-römischen Rechtsgeschichte $^{2}$. Denn es waren weder die literaturgeschichtlich insulare Stellung des 741 promulgierten isaurischen Gesetzbuchs zwischen der justinianischen Kodifikation und deren Wiederbelebung unter den Makedonen noch die formale Originalität der Ecloga als Prototyp des byzantinischen Rechtsbuchs, die ihn veranlaßten, der Ecloga epochalen Rang einzuräumen, vielmehr glaubte er, eine Neugestaltung des Rechts erkennen zu können, die er insbesondere im Strafrecht und mehr noch im Eherecht lokalisierte. In der Behandlung von Konkubinat, Scheidung und mehrfacher Wiederverheiratung durch die Ecloga sah er die christliche Anschauung der Ehe verwirklicht, im Güterrecht fand er „den Gedanken ausgebildet, dass durch und während der Ehe eine Einheit nicht nur der Personen, sondern auch des Vermögens - eine Gütergemeinschaft - entstehe ${ }^{\text {"3 }}$.

Sodann führte Zachariās unumstrittene Autorität dazu, daß sein Urteil vielfach ohne ernsthafte Prüfung übernommen wurde ${ }^{4}$. Auch früh geäußerter Widerspruch

1 Karl Eduard Zachariä von Lingentbal, Geschichte des griechisch-römischen Rechts (Berlin ${ }^{3} 1892$ [Ndr. Aalen 1955]) 84, 89. Die beiden angeführten wie auch alle folgenden Zitate finden sich bereits in der noch unter anderem Titel erschienenen 1. Auflage (1. Heft Leipzig 1856) und selbstverstāndlich auch in der 2. Auflage (Berlin 1877).

${ }^{2}$ Kritisch rekonstruiert von Dieter Simon, Die Epochen der byzantinischen Rechtsgeschichte, in: Ius Commune 15 (Frankfurt am Main 1988) 73-106 (hier 73-88).

3 Zacbariä, Geschichte, 89, vgl. auch 57, 58, 78-79 und 82.

4 Ohne Anspruch auf Vollständigkeit und ohne Berücksichtigung unterschiedlicher Nuancierungen seien genannt Jos. Zhishman, Das Eherecht der orientalischen Kirche (Wien 1864)

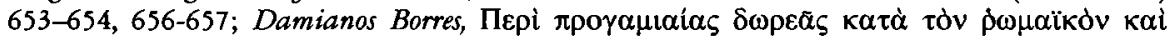

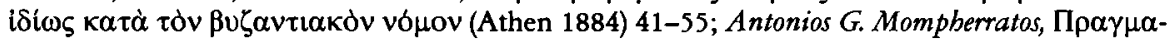

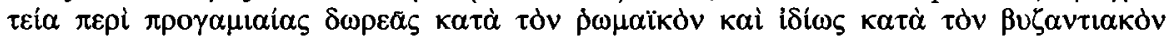
vóuov (Athen 1884) 67-70; Demostbenes D. Desminis, Die Eheschenkung nach römischem und insbesondere nach byzantinischem Recht (Athen 1897) 16-30; Franfois Dupouy, Le Droit civil Romain d'après l'Ecloga (Bordeaux 1902) 72-81; Edwin Hanson Freshfield, A Manual of Roman Law. The Ecloga (Cambridge 1926) 17-19, 24-25; N. P. Blagoev, Ekloga (Sofia 1932) 26-27, 47- 
zum zentralen Punkt der Gütergemeinschaft ${ }^{5}$ konnte nicht verhindern, daß noch in jüngerer Zeit die inhaltliche Andersartigkeit des isaurischen Ehegüterrechts als selbstverständlich angenommen und mit dem Einfluß griechischen Volksrechts erklärt ${ }^{6}$ oder in den „Übergang der oströmischen Sklavenhaltergesellschaft in den byzantinischen Frühfeudalismus“ eingepaßt wurde ${ }^{7}$. Andererseits hat es auch an energischen, die materielle Neuartigkeit der Ecloga global negierenden Protesten nicht gefehlt. So gab H. J. Scheltema zu Protokoll: „... namentlich das Ehegüterrecht - das nebenbei gesagt keine Spur irgendeiner Gütergemeinschaft aufweist - entspricht, soweit ich sehe, dem, was aus der justinianischen Gesetzgebung hervorgeht. ${ }^{\text {"8 }}$ Und kürzlich hat sich Dieter Simon in ähnlicher Weise geäußert: „... sei es die glatte Erfindung einer durch die Ecloga angeblich unter Ehegatten und Kindern eingeführten Gütergemeinschaft ...- durchgängig stellt sich bei einer sorgsamen Vergleichung der Vorschriften der Ecloga mit älteren Gesetzen oder Rechtszuständen heraus, daß Zachariaes Bilderstürmer-Normen entweder nicht existieren oder schon justinianisch sind oder lediglich bereits für frühere Epochen erschließbare Gewohnheiten erstmals formulieren." "9

Ein „Forschungsstand“ läßt sich aus diesem Meinungsbild schwerlich konstruieren. Man wird unterstellen dürfen, daß Scheltema die rhetorisch angemahnte „genaue Untersuchung“ und Simon die behauptete „sorgsame Vergleichung“ vorgenommen haben - zu einer Vorführung ihrer Interpretationen hatten beide keinen Anlaß. Die folgenden Bemerkungen versuchen, das für den Bereich des Ehegüterrechts nachzuholen und sich dabei nicht auf eine vergleichende Untersuchung der dogmatischen Strukturen zu beschränken, sondern auch die formale Präsentation des Rechtsstoffs durch die Ecloga zu berücksichtigen, die nicht zuletzt durch den Auswahlcharakter dieses Gesetzbuchs geprägt ist.

Entsprechend dem im Prooimion (Z. 45-46 ${ }^{10}$ ) ausgedrückten Streben nach Knapp-

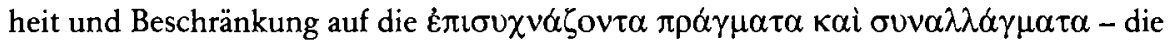
häufig begegnenden Angelegenheiten und Verträge - läßt die Ecloga manche in der justinianischen Kodifikation präsenten oder gar breit traktierten Regelungskomplexe

49. Zurückhaltender die Äußerungen von E. E. Lip̌sic, Ėkloga. Vizantijskij zakonodatel'nyj svod VIII veka (Moskau 1965) 88-92.

5 Vgl. unten Anm. 44.

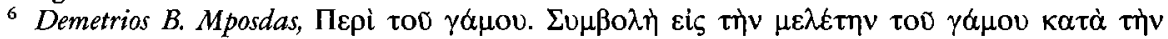

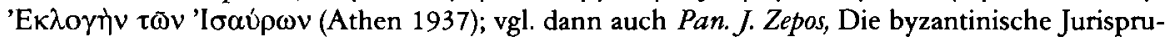
denz zwischen Justinian und den Basiliken, in: Berichte zum XI. Internationalen ByzantinistenKongreß (München 1958) Nr. V,1, S. 14-16.

7 Heinz Herz, Von der Justinianischen Kodifikation zur Ekloge. Rechtshistorische Anmerkungen zum Übergang der oströmischen Sklavenhaltergesellschaft in den byzantinischen Frühfeudalismus, in: Joachim Hermann, Helga Köpstein und Reimar Müller(Hrsg.), Griechenland - Byzanz - Europa (Berlin ${ }^{2}$ 1988) 81-92.

${ }^{8}$ Im Korreferat zu Zepos' oben Anm. 6 zitiertem Bericht, ebenda (Korreferate) 40; vgl. dann auch $N$. van der Wal/J. H. A. Lokin, Historiae iuris graeco-romani delineatio (Groningen 1985) 72.

9 Simon, Epochen (wie oben Anm. 2), 83-84.

10 Alle Zeilen- und Kapitelangaben beziehen sich auf die 1983 in Frankfurt am Main erschienene Ausgabe. 
des Ehegüterrechts völlig unerwähnt. So ist zum Beispiel vom Besteller der Mitgift lediglich an zwei Stellen (Z. 301 und 354) und dort nur inzident die Rede, eine Differenzierung zwischen dos profecticia und dos adventicia wird nicht angesprochen. Untraktiert bleibt ferner die Unterscheidung zwischen geschätzter und ungeschätzter Mitgift; und - um ein drittes Beispiel zu nennen - die Verwaltung der Mitgift während bestehender Ehe - Stichworte etwa: Nießbrauch, Vermögensverfall des Ehemannes, Veräußerung von Dotalgrundstücken - wird nicht im Detail geregelt. Schließlich sei noch an eine Selbstverständlichkeit erinnert: Wie in der gesamten Ecloga ist auch im Ehegüterrecht die Nomenklatur der Klagarten verschwunden ${ }^{11}$.

Zwei potentielle Konfliktfälle erschienen den Verfassern der Ecloga immerhin wichtig genug, daraus einen eigenen Titel 3 zu machen: die dos non numerata und das privilegium dotis ${ }^{12}$.

Im 1. Kapitel wird dem Ehemann für den Fall einer schriftlich oder mündlich vereinbarten - also versprochenen und quittierten -, aber nicht übergebenen Mitgift für Forderung bzw. Einrede eine Frist von fünf Jahren zugebilligt, die mit der EheschlieBung zu laufen beginnt - bei Minderjährigkeit des Ehemannes mit der Erreichung der Volljährigkeit. Das entspricht der vorjustinianischen Regelung der exceptio bzw. querela non numeratae pecuniae ${ }^{13}$. Justinian hatte dafür die Verjährung auf zwei Jahre herabgesetzt (C. 4.30 .14 pr. [a.528]); für die Mitgift hatte er Sonderregelungen eingeführt, indem er die Frist einerseits erst mit der Auflösung der Ehe beginnen ließ, andererseits verkürzte - zunächst auf ein Jahr (C. 5.15 .3 [a.528]), später dann in Abhängigkeit von der Ehedauer noch weiter (N. 100 [a.539]). Die Isaurer sind in diesem Fall sicherlich nicht zufällig zum vorjustinianischen Rechtszustand zurückgekehrt ${ }^{14}$. Über ihr Motiv kann nur spekuliert werden, zumal die Ecloga eine allgemeine Regel über die non numerata pecunia nicht enthält. $\mathrm{Zu}$ notieren ist aus diesem Kapitel schließlich, daß auch in der Ecloga den Eltern der Frau das beneficium competentiae gewährt wird $^{15}$.

Kapitel 2 sichert den Anspruch der Frau auf Herausgabe der Mitgift: Nach dem Tode des Mannes sollen weder der Fiskus noch private Gläubiger auf das Vermögen zugreifen dürfen, bevor die Frau ihre Mitgift zurückerhalten hat. Die Formulierung läßt - anders als bei Justinian - den Gedanken an ein (stillschweigendes General-) Pfand nicht erkennen und scheint das Konkursprivileg auf die Konkurrenz mit später entstandenen Forderungen zu beschränken ${ }^{16}$, wobei die ausdrückliche Erwähnung

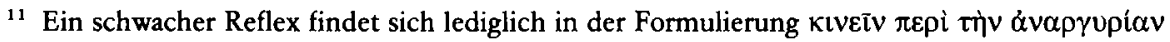
(Z. 307).

${ }_{12}$ Vgl. zu diesem Titel zuletzt Fausto Goria, Tradizione romana e innovazioni bizantine nel diritto privato dell'Ecloga privata aucta. Diritto matrimoniale (Frankfurt am Main 1980) 118-133.

${ }^{13}$ Vgl. Max Kaser, Das römische Privatrecht (im folgenden Kaser, RPR) I (München ${ }^{2} 1971$ ) $542^{39}$.

${ }_{14}$ Ein ununterbrochenes Fortleben in der Rechtspraxis braucht man dabei nicht vorauszusetzen, da die alte Frist von fünf Jahren nicht nur in der Konstitution von 528, sondern auch an zwei Stellen der Institutionen (3.21 und 4.13.2) erwähnt wird.

${ }_{15}$ Zum klassischen und justinianischen Recht vgl. Kaser, RPR I 482 ${ }^{\mathbf{4 2 , 4 3}}$ und II (München $\left.{ }^{2} 1975\right) 187^{19}, 331^{15}$.

${ }^{16}$ Insofern ist es vielleicht doch nicht ganz beliebig, wenn die Ecloga in der Rubrik des Titels 
von Fiskalforderungen freilich nur vor dem Hintergrund der einschlägigen justinianischen Bestimmungen ${ }^{17}$ zu verstehen ist.

Materiell ist diese Norm dem 3. Kapitel des Titels 10 über das Darlehen benachbart. Dort wird die Haftung der Mitgift für (Darlehens-)Schulden des - an dieser Stelle wohl als noch lebend vorausgesetzten - Mannes ausgeschlossen. Eine Ausnahme soll gelten, wenn die Frau sich freiwillig mitverpflichtet hat. Justinian hatte dies in $\mathrm{N}$. 134.8 (a.556) ausdrücklich als ungenügend bezeichnet und eine Ausnahme nur für den Fall zugelassen, daß es sich um eine Aufwendung zugunsten der Frau gehandelt habe.

Unabhängig davon, ob diese wohl unstrittigen Abweichungen vom justinianischen Recht rechtspolitischer Absicht oder dem Streben nach dogmatischer Vereinfachung entspringen, haben sie eher marginalen Charakter und sind auch von Zachariä keineswegs als Belege für das angeblich eigenständige System der Ecloga in Anspruch genommen worden.

Dieses lokalisierte er vielmehr in Titel 2, der „über erlaubte und verbotene Ehen, erste und zweite, schriftliche und nichtschriftliche, und deren Auflösung " handelt. Der Titel beginnt mit der Nennung der Ehevoraussetzungen, woran sich die Aufzählung der Ehehindernisse anschließt. Es folgt ein Komplex über die als idealtypisch angesehene Ehe mit schriftlicher Vereinbarung: Kapitel 3 bespricht den Ehevertrag, die beiden folgenden Kapitel regeln die güterrechtlichen Konsequenzen. Ein nächster Komplex aus zwei Kapiteln behandelt dann die Ehe ohne schriftliche Vereinbarung. Daran schließen sich in Kapitel 8 Bestimmungen zur Zweitehe an. Den Abschluß bildet das Scheidungsrecht (Kapitel 9), das durch ein eigenes Prooimion eingeleitet wird.

Während dieser einleuchtend gegliederte Aufbau des Titels exemplarisch eine der Stärken der Ecloga verdeutlicht ${ }^{18}$, zeigt das den ehegüterrechtlichen Teil eröffnende Kapitel 3 ebenso beispielhaft die Schwierigkeiten der Verfasser, komplexe Regeln knapp und klar zu formulieren. Dabei ist einzuräumen, daß manche Unklarheiten nur für den heutigen Interpreten bestehen, der nicht über das als selbstverständlich vorausgesetzte Praxiswissen des zeitgenössischen Adressaten verfügt. Ungeschickt wenn auch letztlich irrelevant - ist es jedenfalls, daß zunächst von einer Zusatzklausel in der vom Mann ausgestellten Urkunde gesprochen wird, dann aber von einer zusätzlichen dritten Urkunde. Überhaupt fällt auf, daß nur von den Verpflichtungen des Mannes die Rede ist. Dieser muß zunächst den vollständigen Empfang der Mitgift bestätigen ${ }^{19}$ und deren ungeminderte Bewahrung zusagen, wobei Bestätigung und Zusage sich auch auf das erstrecken soll, was der Mann der Frau zur Erhöhung der Mitgift etwa hinzugeschenkt hat. (Diese Vermögensmasse bezeichne ich im folgenden als

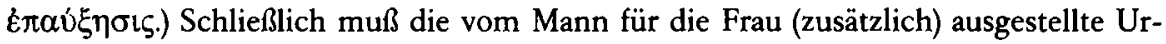

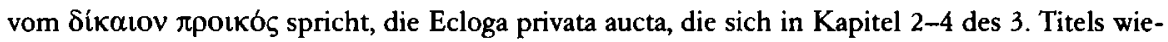

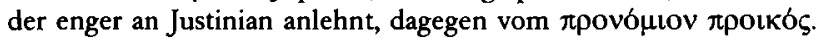

${ }^{17} \mathrm{Zu}$ diesen vgl. Kaser, RPR II, 192-193.

18 Man vergleiche zu diesen Gliederungsfragen auch das Zeugnis der zwar wahrscheinlich nicht authentischen, aber vermutlich (fast) zeitgenössischen Kapitelindices (Pinakes; hier Z. 13-35).

${ }_{19}$ Wie sich aus E. 3.1 ergibt, muß er dies auch dann, wenn die Mitgift noch nicht (oder nicht vollständig) übergeben worden ist. 
kunde eine Vereinbarung für den Fall der Kinderlosigkeit enthalten. Hier hängen die Wörter $\mu \varepsilon \rho \circ \varsigma \tau \varepsilon \tau \alpha \rho \tau o v(Z .160)$ syntaktisch in der Luft, lassen sich auch als accusativus limitationis kaum rechtfertigen, könnten daher als Glossem verdächtigt werden. Überdies wird zunāchst nicht mitgeteilt, auf welche Bezugsgröße sich die Quote von einem Viertel beziehen soll.

Der letzte Satz des Kapitels erscheint ebenfalls nicht ganz eindeutig. Bei unvoreingenommener Lektüre ist die Verneinung des imperativischen Infinitivs jedoch eher als Ausdruck eines Verbots als der Befreiung von einer Verpflichtung aufzufassen und

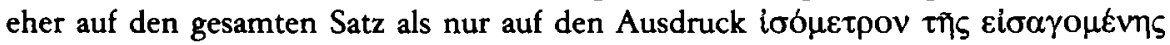

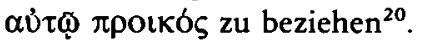

Die folgenden Kapitel entfalten eine ganz elementare Kasuistik. Als Eheauflösungsgrund wird allein der Tod eines Ehepartners vorausgesetzt, das mögliche Vorhandensein von Enkeln, d.h. Kindern vorverstorbener Kinder, bleibt unerwähnt; ebensowenig werden Regelungen für den Fall getroffen, daß Kinder nach Auflösung der Ehe, aber noch zu Lebzeiten des verwitweten Elternteils versterben ${ }^{21}$. In der Ecloga werden nur drei paarige Diäresen unterschiedlichen Ranges abgehandelt: 1. Sind Kinder vorhanden oder nicht? 2. Geht der überlebende Ehepartner eine neue Ehe ein oder nicht? 3. Verstirbt der Mann oder die Frau? Von den acht möglichen Konstellationen werden in den Kapiteln 4 und 5 zunächst nur sechs geregelt. Der Aufbau der beiden Kapitel läßt sich durch das folgende Schema verdeutlichen:

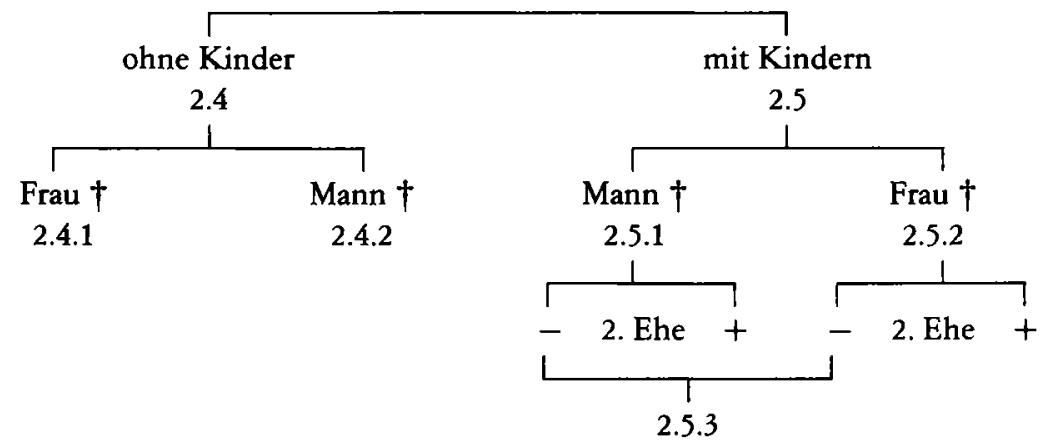

Zunächst behandelt Kapitel 4 den Fall der kinderlosen Ehe: Verstirbt die Frau, so soll der Mann ein Viertel der Mitgift erhalten. Umgekehrt soll bei Vorversterben des Mannes die Frau zusätzlich zur Rückerstattung der Mitgift noch ein Viertel des Mit-

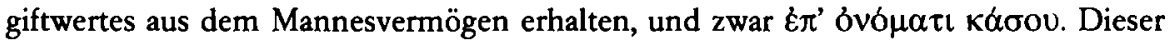
$z w e i t e$ Teil stützt das $\mu \ell \rho \circ \varsigma \tau \varepsilon \tau a \rho \tau o v$ in Kapitel 3 entscheidend. Das bedeutet, daß

\footnotetext{
${ }^{20}$ Dies ergibt sich vor allem aus der Stellung der Verneinungspartikel. Wollte man die Vernei-

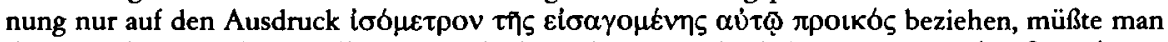

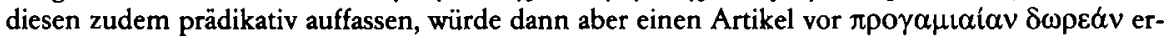
warten.

${ }^{21} \mathrm{Vgl}$. dagegen etwa die entsprechenden Regelungen in den Novellen 2 und 22 sowie die Ausführlichkeit, mit der Justinian die güterrechtlichen Folgen einer Scheidung behandelt.
} 


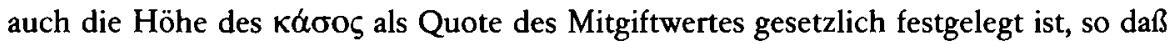
die dritte, vom Mann zu errichtende Urkunde ausschließlich dazu dient, die Vollstrekkung der $\kappa \alpha \sigma o \varsigma$-Forderung seitens der Frau zu erleichtern ${ }^{22}$.

Was bei Vorhandensein von Kindern zu geschehen hat, steht in Kapitel 5:

Stirbt der Mann, so fällt der Besitz von Mitgift und Mannesvermögen an die Frau; ihr obliegt die Verwaltung des gesamten Familienvermögens, wobei sie als erstes ein öffentliches Inventar aufstellen muß, welches ihre Mitgift und die nachgewiesenermaBen noch vorhandenen $\dot{\varepsilon} \xi \hat{\omega} \pi \rho \circ \kappa \alpha$ gesondert ausweisen muß. Sollte die Frau sich wieder verheiraten, so fällt das gesamte Vatergut an die Kinder, die ihrer Mutter ledig-

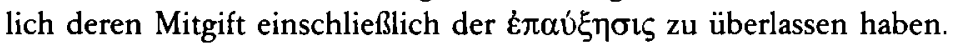

Stirbt hingegen die Frau, so soll der Mann Besitzer der Mitgift und der $\xi \xi \hat{\xi} \pi \rho \circ \kappa \alpha$ sein und die Verwaltung des gesamten Familienvermögens führen. Das gilt auch bei seiner Wiederverheiratung; erst wenn die Kinder volljährig sind, muß er ihnen ihr Muttergut ungemindert herausgeben.

Die Regelungen dieser beiden parallelen Teile sind materiell nicht spiegelbildlich. Die Inventarerrichtung wird nur der Frau auferlegt; was im Fall der $\delta \varepsilon v \tau \varepsilon \rho \circ \gamma \alpha \mu \circ \bar{\sigma} \sigma \alpha$ geschieht, wenn ihre Kinder aus erster Ehe noch minderjährig sind, bleibt vorläufig ungeregelt. Dieses inhaltlich begründete Ungleichgewicht wird formal dadurch ausgeglichen, daß das im ersten Teil nur erwähnte göttliche Gebot im zweiten Teil ausgeschrieben wird. Daß dies rhetorische Absicht ist, zeigt sich auch daran, daß die beiden Abschnitte chiastisch zu den beiden Teilen von Kapitel 4 stehen und daß in beiden Kapiteln, besonders aber in Kapitel 4 vom Stilmittel der variatio reichlich Gebrauch gemacht wird.

In einem dritten Abschnitt des Kapitels 5 geht es schließlich um die Auflösung der familiären Haushaltsgemeinschaft ohne Wiederverheiratung des überlebenden Elternteils. Sie wird erst dann gestattet, wenn die Kinder die Volljährigkeit und die Möglichkeit eigener Haushaltsführung erreicht haben. Der überlebende Elternteil erhält in diesem Fall außer dem eigenen Vermögen noch einen Kindesteil aus dem Vermögen des verstorbenen Ehegatten.

Die Kapitel 6 und 7 sind in der sog. „Ecloga privata“ zu einem einzigen zusammengefaßt worden. Das entspricht der durchgängig zu beobachtenden, bisweilen übertriebenen Tendenz dieser Eclogarezension, Gliederungsprobleme durch die Bildung größerer Einheiten zu entschärfen. Im vorliegenden Fall besteht das Problem darin, daß der zweite Teil von Kapitel 6 und das Kapitel 7 Sonderfälle der ohne Schriftform geschlossenen Ehe regeln, deren allgemeine Grundsätze im ersten Teil von Kapitel 6 dargelegt werden. Nach diesen Grundsätzen bildet die schriftlose, d.h. ohne schriftlichen Mitgiftvertrag geschlossene Ehe in systematischer Hinsicht die Ausnahme, ist dennoch gültig, sofern eine redliche Übereinkunft der Partner und ihrer Eltern vorliegt und die Eheschließung in öffentlicher religiöser Zeremonie oder unter Publikation im Freundeskreis erfolgt. „Aber auch“ - und hier beginnt der gleitende Übergang zum ersten Sonderfall - ,jeder, der mit einer freien Frau zusammenwohnt, ihr die Be-

22 So schon Francesco Brandileone, Sulla storia e la natura della „donatio propter nuptias“ (Bologna 1892) 46 (Ndr. in: Ders., Scritti di storia del diritto privato italiano. I (Bologna 1931) 117$\left.214\left[155^{3}\right]\right)$. 
sorgung seines Hauses anvertraut und sich fleischlich mit ihr verbindet, geht eine nichtschriftliche Ehe mit ihr ein.“ War also - wie Zachariä von Lingenthal meinte „nach der Ecloga der Bilderstürmer Leon und Constantin, in welcher indessen einmal der Ausdruck $\pi \alpha \lambda \lambda \alpha \kappa \eta \dot{~ v o r k o m m t, ~ . . . ~ j e d e r ~ C o n c u b i n a t ~ o h n e ~ W e i t e r e s ~ a l s ~ E h e ~ z u ~ b e-~}$

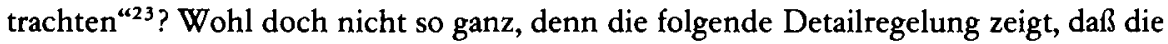
kinderlose Konkubine, anders als die Ehefrau, verstoßen werden durfte, wobei ihr der Mann dann lediglich, falls kein gesetzlicher Scheidungsgrund vorlag, die von ihr eingebrachten Sachen zu erstatten und ein Viertel seines Vermögens zu übergeben hatte.

Auf den Fall der Kinderlosigkeit beschränken sich auch die Regelungen, die in Kapitel 7 bezüglich der vermögenslosen Frau getroffen werden. Stirbt der Mann ohne Testament, soll sie ein Viertel seines Vermögens, jedoch nicht mehr als 10 Pfund er-

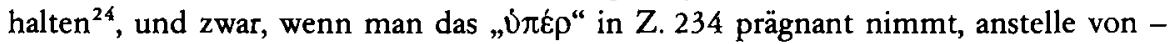

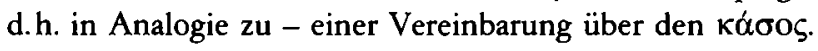

Kapitel 8 faßt die Bestimmungen über die Deuterogamie zusammen, für die dieselben Voraussetzungen gelten wie für eine erste Ehe und deren Eingehung, solange keine Kinder aus der ersten Ehe da sind, völlig problemlos ist ${ }^{25}$. Sind solche Kinder vorhanden, dürfen weder Mann noch Frau dem neuen Ehepartner mehr als einen Kindesteil zuwenden. Die $\delta \varepsilon \cup \tau \varepsilon \rho o \gamma \alpha \mu \circ \sigma \sigma \alpha$ muß bei Gefahr der Infamie eine zwölfmonatige Frist abwarten. Die folgenden Sätze wiederholen die einschlägigen Bestimmungen aus Kapitel 5, Abschnitt 1 und 2 und schärfen ein, daß der wiederheiratende Elternteil nichts aus dem Vermögen des ersten Ehepartners erhalten dürfe ${ }^{26}$. Den Abschluß bilden zwei Ergänzungen. Die erste ist erbrechtlicher Natur: Die Kinder aus erster und zweiter Ehe sollen den jeweils gemeinsamen Elternteil gleichberechtigt beerben $^{27}$. Die zweite Ergänzung holt für die Frau nach, was für den Mann bereits in 2.5.2 geregelt und in 2.8.1 wiederholt worden war: Sind die Kinder aus erster Ehe noch minderjährig, muß die Frau anders als der Mann einen Vormund für sie beantragen.

Damit ist der immanente Durchgang durch den ehegüterrechtlichen Teil des Titels 2 beendet. Bei dem nun fälligen Vergleich mit den justinianischen Bestimmungen empfiehlt es sich, mit den Kapiteln 6-7 zu beginnen, da hier die Unterschiede offenkundig am geringsten sind. Justinian hatte innerhalb von 10 Jahren in nicht weniger als fünf Gesetzen einschlägige Bestimmungen erlassen bzw. wiederholt. Zunächst

\footnotetext{
23 Zacbariä, Geschichte, 58 mit Bezug auf E. 6.7 (13).

24 Nach erneuter Abwägung der Argumente würde ich heute den Satz $\varepsilon \dot{i}-\kappa o \mu i \zeta \varepsilon \sigma \theta \alpha$ l (Z. 235236) in den Apparat verbannen.

${ }^{25}$ Mit dem knappen ersten Satz wird gewissermaßen die noch ausstehende Behandlung der letzten beiden von den oben erwähnten acht Fallkonstellationen nachgeholt.

${ }^{26}$ Gegen Zachariä, Geschichte, 91 mit Anm. 232 und 234, ergibt sich aus diesem Satz nicht, daß

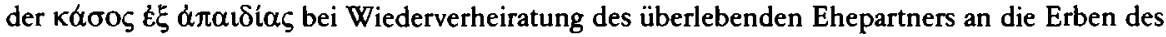
verstorbenen zurückfällt, da sich alle Bestimmungen von E. 2.8.1 von den Worten $\varepsilon \dot{\varepsilon} \delta \dot{\varepsilon} \pi \alpha \tilde{\imath} \delta \varepsilon \zeta$ ü $\pi \varepsilon \iota \sigma \iota(Z .241)$ an auf den Fall des Vorhandenseins von Kindern beziehen. Das entspricht auch der Vorlage (N. 22.22), an die sich die Ecloga hier besonders eng anschließt.

27 Zachariä, Geschichte, $92^{239}$ hatte den außerordentlich lapidar formulierten Schlußsatz

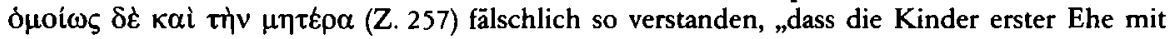
denen der zweiten die zweite Frau ihres Vaters beerben soll( $t$ )en“.
} 
hatte er in C. 5.17 .11 pr.-1a (a.533) und erneut in N. 22.18 (a.535) von der verstoßenen Konkubine gesprochen, dann in N. 53.6 (a.537) von der armen Witwe. In N. 74.5 (a.538) und N. 117.5 (a.542) schließlich hatte er, immer wieder sich selbst zitierend, beide Fälle zusammenfassend behandelt, so daß sich also auch der Redaktor der Ecloga privata bei seiner Zusammenfassung der beiden Kapitel auf das justinianische Vorbild hätte berufen können, während die Autoren der Ecloga bei der Abfassung von Kapitel 6 die Konstitution vom Jahre 533 oder deren Zitat in Novelle 22, bei Kapitel 7 Novelle 53 vor Augen gehabt haben dürften. Bezogen auf den Fall der Kinderlosigkeit unterscheiden sich die justinianischen Bestimmungen untereinander überhaupt nicht, von der Ecloga lediglich in einem Punkt: Die Höchstgrenze, die für das Viertel der verstoßenen Konkubine gelten soll, wird von Justinian bei 100 Pfund gezogen, liegt also - nominal! - zehnmal so hoch wie in der Ecloga ${ }^{28}$.

Auch in den güterrechtlichen Regeln zur Wiederverheiratung folgt die Ecloga materiell soweit als möglich den Normen der justinianischen Kodifikation. So findet sich das Verbot, dem neuen Ehepartner aus dem eigenen Vermögen mehr als den Teil eines Kindes aus der ersten Ehe zuzuwenden, von Justinian zweimal wiederholt, bereits in einer Konstitution Leons $\mathrm{I}^{29}$, und auch das gemeinsame Erbrecht von Kindern aus verschiedenen Ehen nach dem jeweils gemeinsamen Elternteil stellt keine Neuerung dar ${ }^{30}$.

Es bleibt somit der Kern des ehelichen Güterrechts zu betrachten, nämlich Herkunft und Zuordnung der Vermögensbestandteile in der Regelehe sowie ihr Schicksal nach deren Auflösung. Unmittelbar kritisch ist dabei der Beitrag von Mannesseite, der deshalb auch in der Literatur besonders ausgiebig traktiert worden ist. Ausgangspunkt der Erörterung ist gewöhnlich der Schlußsatz von E. 2.3, in dem Justinians Vorschrift negiert wird, der Mann müsse der Frau eine der Mitgift gleichwertige $\pi p o \gamma \alpha \mu \iota \alpha i \alpha$ $\delta \omega \rho \varepsilon \alpha$ bestellen. Da dieser Ausdruck an anderer Stelle der Ecloga nicht mehr verwendet wird, stellt sich die Frage, wieweit und wodurch die justinianische $\pi \rho 0 \gamma \alpha \mu 1 \alpha i \alpha$ $\delta \omega \rho \varepsilon \alpha$ in der Ecloga vertreten wird. Dazu ist zunächst der justinianische Status in aller Kürze zu rekapitulieren ${ }^{31}$ : Nach justinianischem Recht war die $\pi \rho 0 \gamma \alpha \mu \iota \alpha i \alpha$ $\delta \omega \rho \varepsilon \alpha$ der Beitrag des Mannes zum Ehevermögen, der vor der Eheschließung ausgehandelt und im Ehevertrag festgelegt wurde. Während der Ehe unterlag die $\pi \rho \circ \gamma \alpha-$

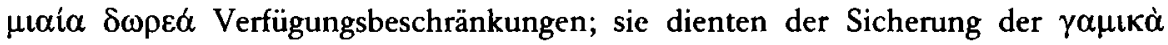
$\kappa \hat{\varepsilon} \rho \delta \eta$, welche für den Fall der - hier durch den Mann verursachten - Auflösung der Ehe frei vereinbart werden konnten. Da Justinian gleichzeitig in eher verstärktem Maße dafür sorgte, daß $\pi \alpha \tau \rho థ \alpha$ und $\mu \eta \tau \rho \Phi \alpha \pi \rho \alpha \gamma \mu \alpha \tau \alpha$ für die Kinder bewahrt wurden, entstand ein eigenes $\kappa \hat{\varepsilon} \rho \delta$ o $\varsigma$ für den überlebenden Ehepartner nur im Falle der

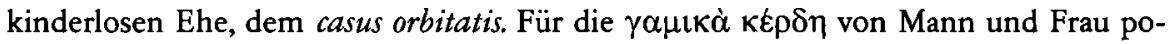
stulierte Justinian Äquivalenz. Da er offenbar die schon traditionelle Identität der

${ }^{28}$ Diese Herabsetzung der Obergrenze durch die Isaurer dürtte wohl eher auf der Veränderung der allgemeinen wirtschaftlichen Verhāltnisse als auf einer absichtlichen Schlechterstellung der Konkubine beruhen.

29 C. 5.9 .6 pr. (a.472); C. 5.9 .9 (a. 529); N. 22.27 (a.536).

30 Vgl. z. B. N. 22.29 (a.536).

31 Vgl. dazu Kaser, RPR II, 193-201 mit Literatur. 
Quoten nicht aufgeben wollte, mußte er die Gleichwertigkeit von $\pi \rho \circ \gamma \alpha \mu \iota \alpha i \alpha \delta \omega \rho \varepsilon \alpha$ und Mitgift zur Regel machen. Damit war die $\pi \rho \circ \gamma \alpha \mu \iota \alpha i \alpha \delta \omega \rho \varepsilon \alpha$ zum Spiegelbild der Mitgift geworden.

Die Ecloga kennt ebenfalls eine bei Gelegenheit der Eheschließung erfolgende Gabe des Mannes an die Frau, betrachtet sie aber nicht als obligatorisch - geschweige denn, daß sie ihre relative Höhe vorschreibt - und bezeichnet sie in leicht variierenden Periphrasen als eine Erhöhung der Mitgift ${ }^{32}$. Damit ist die Mannesgabe nicht spiegelbildliches Pendant, sondern integrierender Bestandteil der Mitgift ${ }^{33}$, müßte also deren Schicksal in jedem Fall teilen. Zur Vergewisserung gibt es dennoch Anlaß. Er liegt weniger darin, daß die justinianische Mannesgabe trotz ihrer Bezeichnung als $\delta \omega \rho \varepsilon \alpha ́$ keineswegs zum Frauenvermögen gerechnet wurde, als in dem Befund, daß in der Ecloga die દ̇ $\pi \alpha u ́ \xi \eta \sigma \iota \varsigma$ bisweilen, aber nicht immer ausdrücklich neben der Mitgift

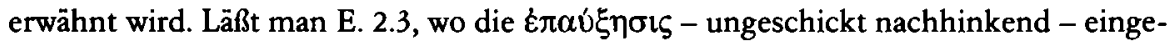
führt wird, außer Betracht, wird sie nur im Fall der wiederheiratenden Mutter explizit angeführt, der in E. 2.5.1 und 2.8.1 materiell übereinstimmend geregelt ist. Die Formulierungen in diesen beiden Kapiteln lassen keinen Zweifel daran, daß die

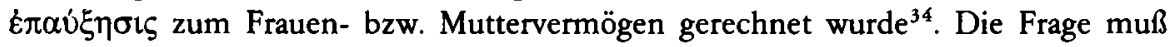

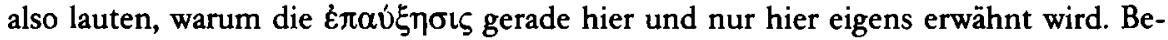
zogen allein auf den Fall des Vorhandenseins von Kindern, leuchtet der Unterschied zwischen den Abschnitten 1 und 2 des Kapitels 5 sofort ein: Denn wenn die $\varepsilon \tilde{\varepsilon} \alpha u ́ \xi \eta \sigma \iota \zeta$ als Schenkung des Mannes an die Frau auch sofort in das Frauenvermögen übergeht und damit Bestandteil der $\mu \eta \tau \rho \oint \alpha \pi \rho \alpha \gamma \mu \alpha \tau \alpha$ wird, stammt sie doch ursprünglich aus dem Vermögen des Mannes bzw. Vaters, so daß im Falle von dessen Tod ihr Verbleiben bei der Mutter kontraintuitiv wirken könnte. Daß in Kapitel 5 das Ungleichgewicht in der Erwähnung nicht zufällig ist, zeigt sich auch daran, daß (fast) reziprok die $\hat{\varepsilon} \xi \hat{\omega} \pi \rho \circ \kappa \alpha$, deren durchgängige Zugehörigkeit zum Frauenvermögen außer Diskussion steht, in Abschnitt 1 nur im Rahmen der Bestimmung über die Inventarerrichtung auftauchen, während in Abschnitt 2 ausdrücklich gesagt wird, daß der verwitwete Vater in den Besitz der Mitgift und der $\dot{\xi} \xi \omega ́ \pi \rho \circ \kappa \alpha$ gelangt.

Warum die Verfasser der Ecloga es in Kapitel 4 überhaupt nicht für nötig gehalten

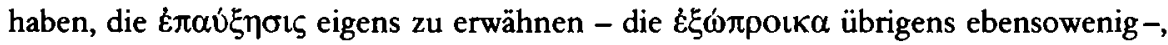
muß dahinstehen. Angesichts der eindeutigen Formulierungen in E. 2.5.1 und 2.8.1

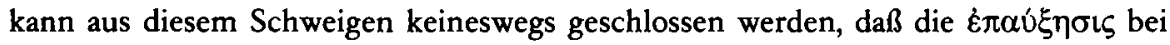
Auflösung einer kinderlosen Ehe vor einer Berechnung des $\gamma \alpha \mu \iota \kappa \delta \nu \kappa \varepsilon \rho \delta \circ \zeta$ an den

32 Z. 158, 193-194 und 250-251.

33 Brandileone, Sulla storia (wie oben Anm. 22), 31-51 (bzw. 143-159) hat darin eine ungebrochene Tradition der "donatio in dotem redacta" gesehen.

34 Hinsichtlich Kapitel 5 hat schon Henry Monnier, La novelle XX de Léon le Sage, in: Mélanges Fitting (Montpellier 1908 = Aalen/Frankfurt am Main 1969) 121-160 (hier 1394), darauf hinge-

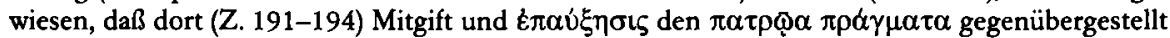
werden. In 2.8.1 ergibt sich dasselbe indirekt aus der Verbindung der beiden Sätze Z. 248-251 und Z. 251-252. 
Mann bzw. dessen Erben zurückfiel ${ }^{35}$. Denn dazu müßte man annehmen, daß die $\dot{\varepsilon} \pi \alpha u ́ \xi \eta \sigma \iota \varsigma$ nach der Vorstellung der Eclogaverfasser erst sobald und nur solange zum Frauenvermögen gerechnet wurde, als überlebende gemeinsame Kinder da waren. Darüber hinaus ist eine feine Differenzierung zu registrieren: Während in E. 2.5.1, wo

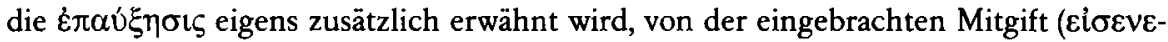
$\chi \theta \varepsilon i \bar{\sigma} \alpha \pi \rho{ }^{\prime} \xi$, Z. 192-193) die Rede ist, wird in beiden Abschnitten des Kapitels 4 von der quittierten Mitgift (ó $\mu \circ \lambda \circ \gamma \eta \theta \varepsilon \tilde{\tau} \sigma \alpha \pi \rho o i \xi \xi$, Z. 165-166 und 171) gesprochen und zu quittieren hatte der Mann nach Kapitel 3 die Mitgift einschließlich der

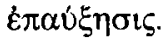

Als Berechnungsgrundlage für den $\kappa \alpha \sigma \sigma o \zeta^{36}$ war die fakultative, in die Mitgift integrierte Mannesgabe nun allerdings unbrauchbar - jedenfalls dann, wenn an der traditionellen Berechnungsweise festgehalten werden sollte. Tatsächlich hat die Ecloga den Modus identischer Quoten beibehalten, die Quote des Kó $\sigma 0 \varsigma$ aber ohne den Umweg über eine Gegenmitgift ebenfalls unmittelbar an die Mitgift gebunden. Damit war wie bei Justinian die Äquivalenz der $\gamma \alpha \mu \iota \kappa \dot{\alpha} \kappa \varepsilon \rho \delta \eta$ für Mann und Frau gewährleistet zumindest dann, wenn der Mann von einer Aufstockung der Mitgift abgesehen hatte. Andernfalls gewann die Frau bei Vorversterben des Mannes aus dessen ursprünglichem Vermögen außer dem Viertel des Werts der von ihr eingebrachten Mitgift nicht nur die $\dot{z} \pi \alpha u ́ \xi \eta \sigma \iota \varsigma$ selbst, sondern auch noch ein Viertel von deren Wert, während andererseits der Mann bei Vorversterben der Frau eine reale Einbuße zugunsten von deren Erben erlitt, sofern er die Mitgift um mehr als ein Drittel aufgestockt hatte. Obwohl man sicher auch für das 8. Jahrhundert annehmen kann, daß der Übergang größerer Vermögensmassen von einer Familie in eine andere vermieden werden sollte ${ }^{37}$, bieten auch die erwähnten Konsequenzen keinen Grund, an der sofortigen und unwiderrufli-

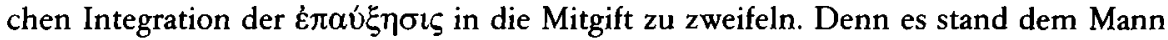
ja frei, ob er die Mitgift überhaupt aufstocken wollte, und der explizit fakultative

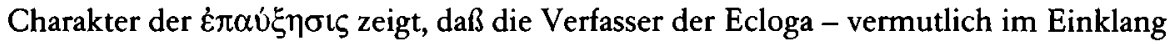
mit herrschenden Gewohnheiten - von einer im Regelfall eher geringfügigen

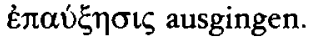

Diese Interpretation des Kapitels 4 wird schließlich auch durch die Eisagoge gestützt. Deren Verfasser nennt zwar die Mannesgabe einerseits (wieder) $\pi \rho 0 \gamma \alpha \mu \iota \alpha i \alpha$

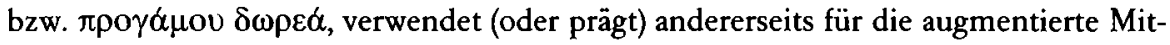

${ }^{35}$ Anders Andreas Schminck unten S. 53 mit Anm. 65.

${ }^{36}$ Zur Entwicklung dieses Instituts vgl. Henry Monnier, Du „casus non existentium liberorum“ dans les novelles de Justinien, in: Mélanges Gérardin (Paris 1907) 437-465. - Es ist symptoma-

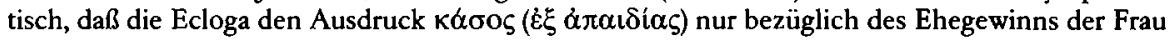
verwendet (Z. 159, 173, 234).

${ }^{37}$ Außerordentlich illustrativ ist in diesem Zusammenhang die Argumentation Leons VI. im Prooimion seiner Novelle 20 (81/5-9 Noailles/Dain). 
gift den Ausdruck $\pi \rho \circ$ Kou $_{\pi}$ ó $\beta o \lambda o v^{38}$, folgt jedoch materiell in wesentlichen Teilen der Kapitel 5 und 8 ihres 19. Titels dem Kapitel 4 des 2. Titels der Ecloga ${ }^{39}$.

Nach der Ecloga lag es also im Belieben des Mannes, den Grad eines etwaigen Ungleichgewichts der $\gamma \alpha \mu \iota \kappa \dot{\alpha} \kappa \hat{\varepsilon} \rho \delta \eta$ zugunsten der Frau zu bestimmen, während die Quote gesetzlich festgelegt war. Justinian dagegen hatte jede Asymmetrie ausgeschlossen, den Vertragspartnern dafür die Möglichkeit gelassen, die Höhe des für beide in gleicher Weise geltenden $\kappa \varepsilon \rho \delta o \zeta$ frei auszuhandeln. Eine gesetzliche Quote hatte Justinian nur für diejenigen Fälle vorgesehen, bei denen es mangels Mitgift auch keine entsprechenden Vereinbarungen gab, wobei als Bezugsgröße dann freilich das Mannesvermögen dienen mußte. Diese Quote von einem Viertel haben die Isaurer wohl kaum zufällig auf die mit Mitgiftvertrag geschlossene Regelehe übertragen ${ }^{40}$.

Der Verzicht auf die $\pi \rho \circ \gamma \alpha \mu \iota \alpha i \alpha \delta \omega \rho \varepsilon \alpha ́ \alpha$ justinianischer Prägung war mithin entgegen Zachariäs Ansicht nicht mit einer Abkehr von dem Isometriegedanken verbunden, und die Ecloga hat den Ausdruck $\pi \rho \circ \gamma \alpha \mu \iota \alpha i \alpha \delta \omega \rho \varepsilon \alpha$ keineswegs deshalb ,geflissentlich vermieden ..., weil sie ... nur an einen freiwilligen Zuschuss zum Ehevermögen Seitens des Mannes gedacht hat “41. Beitrag des Mannes zum Ehevermögen war seine $\pi \varepsilon \rho$ ovoí $\alpha$ schlechthin. Die Frau brachte ihrerseits ihre Mitgift ein, die vom Mann nach Belieben durch eine Schenkung aufgestockt werden konnte. Natürlich wird auch im 8. Jahrhundert das bereits vorhandene, zu erwartende oder (erb-)vertraglich zugesicherte Vermögen des Bräutigams beim Aushandeln einer Ehe eine wesentliche Rolle gespielt haben, und Ehen werden auch zu dieser Zeit vorwiegend zwischen sozial und ökonomisch gleichrangigen Familien vereinbart worden sein. Da die Frau

${ }^{38} \mathrm{Zu}$ diesem Ausdruck, den Zachariä, Geschichte, 91, ohne weiteres auch bei der Beschreibung des „Systems der Ecloga“ für das Ehevermögen verwendet hatte, vgl. Joëlle Beaucamp, Прoเkou-

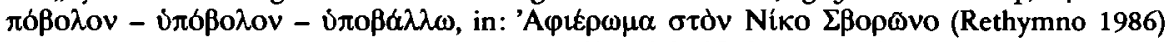

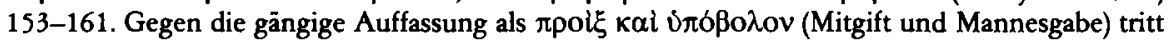
Andreas Scbminck, unten S. 46-54, jetzt für eine Deutung als $\pi \rho 0 \imath \kappa \delta \zeta ~ ¿ \pi \delta \beta 0 \lambda o v$ im Sinne von „Mitgiftwert" ein. Obwohl determinative Komposita auch im Mittelgriechischen unbestreitbar noch wesentlich hāufiger sind als kopulative, scheint mir diese Deutung zu kompliziert, da sie zum einen ein Mißverstāndnis der Eisagoge schon durch Leon VI. (und einen Ecloga-„Scholiasten") annehmen muß und da zum anderen für das Wort úróßo $\lambda o v$ die Bedeutung Mannesgabe in der zweifellos genuinen, auch etymologisch plausiblen synonymen Verwendung zu $\alpha \rho \rho \alpha \beta \omega \dot{v}$

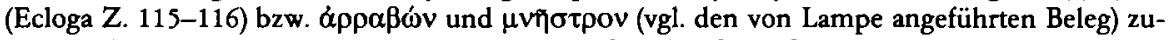
mindest bereits vorbereitet war. Darüber hinaus läßt sich Scbmincks Deutung kaum mit dem Satz

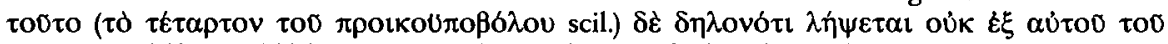

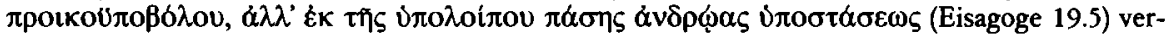

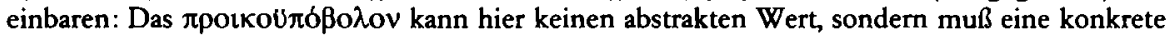
Vermögensmasse bezeichnen; und im zweiten Teil des Satzes liegt der Akzent nicht (nur) auf $\alpha v \delta \rho \omega \alpha \varsigma$, sondern (auch) auf $\vdots \pi 0 \lambda o i \pi 0 v$. Dieser intuitive Bezug auf die ursprüngliche Herkunft des $\dot{\pi} \pi \dot{\beta} \beta 0 \lambda$ ov aus dem Mannesvermögen war für den Verfasser der Eisagoge, der - wie Schminck zeigt - justinianische und isaurische Dogmatik mehrfach unglücklich mischt, besonders naheliegend.

${ }_{39}$ Anders als die Ecloga spricht die Eisagoge allerdings eindeutig von einem Heimfall des $\kappa \dot{\alpha}-$ oo $\zeta$ bei Wiederverheiratung; vgl. oben Anm. 26.

40 Dafür spricht schon der bereits erwähnte Befund, daß sie in E. 2.7 die Quart der armen Witwe

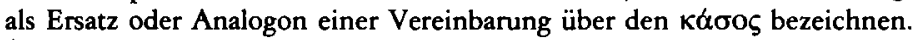

41 Zachariä, Geschichte, 90. 
jedoch regelmäßig dem Hausstand des Mannes folgte, konnten obligatorische Vereinbarungen über den Beitrag des Mannes als überflüssig erscheinen.

Im Falle des Vorhandenseins von Kindern resultierte aus dieser Vereinfachung erst recht kein Unterschied gegenüber Justinian: Hier wie dort übernimmt der überlebende Ehepartner die Verwaltung des Familienvermögens; beim Ausscheiden aus dem gemeinsamen Haushalt erhalten die Kinder ihr Erbe nach dem verstorbenen Elternteil, der überlebende Ehepartner behält einen Kindesteil aus dem Vermögen des verstorbenen, sofern er sich nicht wieder verheiratet ${ }^{42}$. Bei all diesen Regelungen ist es völlig unerheblich, ob ein Teil der $\pi \alpha \tau \rho \Phi \alpha \alpha \rho \alpha \gamma \mu \alpha \tau \alpha$ als $\pi \rho \circ \gamma \alpha \mu \iota \alpha \dot{\alpha} \alpha \delta \omega \rho \varepsilon \alpha$ deklariert war oder nicht.

Erweisen sich somit die realen Konsequenzen der isaurischen Ablehnung einer zur Gegenmitgift ausgestalteten $\pi \rho \circ \gamma \alpha \mu\llcorner\alpha i \alpha \delta \omega \rho \varepsilon \alpha$ als minimal, so bedarf die von Zachariä suggestiv mit der Beobachtung dieser Ablehnung verknüpfte These, nach der Ecloga herrsche „mehr oder minder durchgreifend“ Gütergemeinschaft ${ }^{43}$, erst recht kaum der Widerlegung ${ }^{44}$. Schon die Beibehaltung des Mitgiftsystems, insbesondere der Regelungen, welche die ungeschmälerte Bewahrung der Mitgift sichern, ist mit einer Gütergemeinschaft unvereinbar. Der Mann bleibt Familienoberhaupt und Verwalter des Familienvermögens ${ }^{45}$, an dessen zwar phasenweise latenter, im Auseinandersetzungsfall aber stets aktualisierbarer Scheidung in Mannes- und Frauenvermögen die Ecloga nichts ändert.

Auch zwischen Eltern und Kindern kann von einer Gütergemeinschaft nicht die

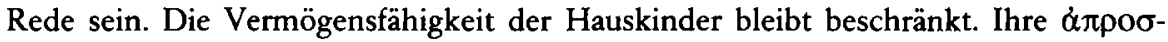

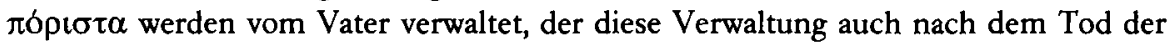
Ehefrau und im Falle seiner Wiederverheiratung in ungebrochener Fortsetzung seiner väterlichen Gewalt fortführt, während das von Justinian gestärkte Sorgerecht der verwitweten Mutter über Person und Vermögen der gemeinsamen Kinder auch in der Ecloga noch vormundschaftliche Züge trägt und im Falle ihrer Wiederverheiratung bei Minderjährigkeit der Kinder durch eine Vormundbestellung abgelöst wird ${ }^{46}$. In allen Konstellationen handelt es sich aber um eine Verwaltung des Vermögens, dessen strikte Trennung in $\pi \alpha \tau \rho \Phi \alpha$ und $\mu \eta \tau \rho \Phi \alpha \pi \rho \alpha \gamma \mu \alpha \tau \alpha^{47}$ im Moment der Auflösung der Haushaltsgemeinschaft auflebt.

\footnotetext{
42 Justinian hatte das in N. 127.3 (a.548) bestimmt.

43 Zacbariä, Geschichte, 90.

$44 \mathrm{Vgl}$. dazu schon V. G. Vasil'evskij, Zakonodatel'stvo ikonoborcev, in: Žurnal Ministerstva Narodnogo Prosveščenija 199 (Okt. 1878) 258-309 und 200 (Nov. 1878) 95-129 (hier 199 [Okt.] 192-293) (Ndr. in: Ders., Trudy. IV [Leningrad 1930] 139-235 [hier 176-178]); Francesco Schupfer, La comunione dei beni tra coniugi e l'Ecloga Isaurica, in: Rivista Italiana per le scienze giuridiche 36 (1903) 319-332; Lujo Margetić, Vizantsko bračno imovinsko pravo u svjetlu novele XX Lava Mudroga, in: Zbornik radova Vizantološkog instituta 18 (1978) 19-50 (hier 24-25).

${ }_{45} \mathrm{Daß}$ dies auch in der Ecloga als selbstverständlich vorausgesetzt wird, zeigt nicht zuletzt das bereits erwähnte Phănomen, daß in E. 2.3 nur die Pflichten des Mannes aus dem Ehevertrag erwähnt werden.

${ }^{46} \mathrm{Zu}$ den justinianischen Bestimmungen s. Kaser, RPR II, 227-228; zur Ecloga vgl. Zachariä(!), Geschichte, 100-101.

47 Zur technischen Verwendung dieser Terminologie durch die Ecloga vgl. auch 16.5.2.
} 
Als Beleg für eine Gütergemeinschaft unter Geschwistern schließlich hatte Zachariä ein Kapitel aus dem Titel über die Pekulien angeführt (E. 16.2) ${ }^{48}$. Diese vorbildlose Bestimmung, die in jüngerer Zeit wegen möglicher Rückschlüsse auf die Militärorganisation im 8. Jahrhundert besondere Aufmerksamkeit gefunden hat ${ }^{49}$, ist in der Tat besonders bemerkenswert, weil sie dogmatische Grundsätze des Pekulienrechts über den Tod des Gewalthabers bzw. der Eltern hinaus fortgelten läßt und eine „Abschreibungsfrist“ für eine aus Familienvermögen erworbene militärische Grundausstattung festsetzt. Im übrigen handelt es sich jedoch um nichts anderes als um eine Auseinandersetzungsregel für eine Erbengemeinschaft unter Brüdern, bei der Kollationspflicht und gleiche Erbteile von jeher die leitenden Prinzipien gewesen waren.

Nahezu all dies hat Zachariä, dem man handwerkliches Unvermögen in puncto rechtshistorischer Hermeneutik wahrlich nicht nachsagen kann, in den Einzelheiten ebenso gesehen. Seine in eklatanter Weise davon abweichende Gesamtbeurteilung wäre trotz ihrer nachhaltigen Wirkung kaum der Rede wert, wenn sie lediglich auf einer eher patriarchalisch geprägten Auffassung von Gleichberechtigung und einer allgemeinen Begeisterung für die Bilderstürmer beruhte. Zachariä scheint darüber hinaus jedoch Opfer der isaurischen Rhetorik geworden zu sein, die die Verfestigung derartiger Vorurteile begünstigte. Der jeweils parallele Aufbau der Kapitel 4 und 5, der

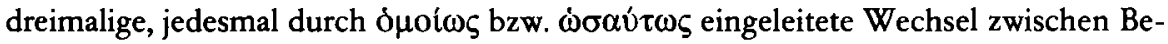
stimmungen, die für den Mann, und solchen, die für die Frau gelten, in E. 2.8.1 sowie die mehrfache Verwendung des Kollektivbegriffs yovei $\varsigma^{50}$ suggerieren eine Gleichstellung der Eheleute. Die Rechte des verwitweten Elternteils am Familienvermögen werden nicht in der "technischeren“ Begrifflichkeit von Eigentum ( $\delta \varepsilon \sigma \pi 0 \tau \varepsilon i \alpha)$, Nutzung $(\chi \rho \tilde{\eta} \sigma \iota \varsigma)$ und Fruchtziehung ( $(\varepsilon\lrcorner \kappa \alpha \rho \pi i \alpha)$ beschrieben, vielmehr ist von „Inneha-

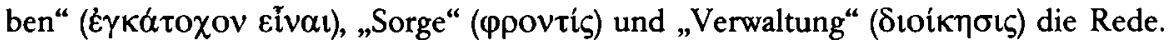
Und schließlich wird an mehreren Stellen die Aufrechterhaltung einer friedvollen Haushaltsgemeinschaft der Familie als regelmäßiger und wünschenswerter, wenn nicht gar obligatorischer Zustand herausgestrichen ${ }^{51}$. Materiell gehen diese Appelle über traditionelle, zum Teil auch bereits gesetzesförmig normierte Eltern- und Kindespflichten nicht hinaus ${ }^{32}$ - Pflichten, auf welche die christliche Lehre keineswegs ein Monopol hatte, zu deren Begründung die Ecloga jedoch unmittelbar, ausschließlich und massiv auf Texte des Alten und Neuen Testaments rekurriert. In dispositiven Partien byzantinischer weltlicher Gesetze scheint diese explizite und zitatgestützte Berufung auf die Bibel ein Novum gewesen zu sein, und die Ecloga macht besonders im Eherecht reichlich davon Gebrauch. Das unterstreicht die bereits durch die Anfangsstellung ausgedrückte Bedeutung, die die Isaurer dem Eherecht zugewiesen haben,

\section{Zachariä, Geschichte, $89^{221}$.}

49 Siehe zuletzt N. Oikonomidès, Middle-byzantine Provincial Recruits: Salary and Armament, in: Gonimos. Neoplatonic and Byzantine Studies Presented to Leendert G.Westerink at 75 (Buffalo 1988) 121-136 (130-134).

${ }^{30}$ Siehe das Wörterverzeichnis s.v., insbesondere Z. 301 und 713 sowie 113,143 und 224. Es erscheint mir sicher, daß es sich hierbei um einen Sprachgebrauch de facto, nicht de iure handelt. ${ }^{31}$ Z. 186-191, 198-208, 212-218 und 713-726.

32 Vgl. Kaser, RPR II, 202-203 und 206. - Welche Rolle hier die Formulierung spielen kann, zeigt etwa der Vergleich von E. 2.5.3 mit dem deutlich nüchterneren Text in Eisagoge 19.8. 
berechtigt jedoch nicht dazu, dieses dem Inhalt nach als besonders christlich zu qualifizieren.

Die materielle Folgenlosigkeit macht die eingesetzte Rhetorik freilich keineswegs funktionslos. Es hat vielmehr den Anschein, als hätten die Verfasser der Ecloga versucht, durch einen klar gegliederten Aufbau, einen eher untechnischen Sprachgebrauch und durch die Berufung auf die Autorität der Bibel die Reduktion dogmatischer Komplexität zu kompensieren. Hatte Justinian, vermutlich höher entwickelte urbane Lebensformen fokussierend, sicherlich aber auch mit einem Überschuß an dogmatischer Phantasie begabt, ein relativ engmaschiges Netz fein differenzierender Regelungen ehegüterrechtlicher Verhältnisse geknüpft, so scheint Leon III., einerseits mit diesem Vorbild, andererseits mit einer sowohl kulturell als auch sozialökonomisch wesentlich schlichteren Realität konfrontiert, den Regelfall familiär-güterrechtlicher Beziehungen zum Idealtypus erhoben zu haben, wobei er es sich dann sogar leisten konnte, in einigen Punkten hinter Justinian zurückzufallen. Formal durchaus originell, erweist sich die Ecloga auf dem Gebiet des Ehegüterrechts damit als in doppeltem Sinne restaurativ. 